\title{
Searches for double Higgs production or decay using the CMS detector
}

\author{
Giacomo Ortona*t \\ Laboratoire Leprince-Ringuet, CNRS \\ Route de Saclay - École Polytechnique \\ 91128 Palaiseau, France \\ E-mail: giacomo.ortona@cern.ch
}

The production of the pairs of Higgs bosons provides a direct handle on the structure of the Higgs field potential. While the HH production within the SM is very small and essentially out of the experimental reach within the Run I or II, several beyond SM theories foresee an enhancement that can be already probed with the available data. These proceedings detail the searches for double Higgs boson production performed at the Large Hadron Collider with the Compact Muon Solenoid experiment. Both resonant and non-resonant productions are discussed using final states in which one of the two Higgs bosons decays in a pair of b quarks, while the other can decay in pairs of $\tau, \mathrm{b}, \mathrm{W}$ or $\gamma$ particles. Exclusion upper limits are set on the resonant and non-resonant double Higgs boson production cross-sections and on the presence of anomalous couplings affecting the non-resonant production.

38th International Conference on High Energy Physics

3-10 August 2016

Chicago, USA

\footnotetext{
*Speaker.

'On behalf of the CMS collaboration. With the support of the P2IO LabEx (ANR-10-LABX-0038) in the framework "Investissements d'Avenir" (ANR-11-IDEX-0003-01) managed by the Agence Nationale de la Recherche (ANR, France).
} 


\section{Introduction}

The measurement of the Higgs boson (h) trilinear coupling, responsible for giving mass to the Higgs boson itsels, is crucial to our understanding of the EWSB mechanism and to explore the presence and properties of any hidden Higgs sector beyond the Standard Model. To this extent, an EFT model with dimension-6 operators is used [1]. In this model, the top-Yukawa coupling $\left(y_{t}\right)$ and the Higgs trilinear coupling $\left(\lambda_{h h h}\right)$ are allowed to vary $\left(k_{\lambda}=\frac{\lambda_{h h h}}{\lambda_{h h h}^{\text {sM }}}, k_{t}=\frac{y_{t}}{y_{t}^{S \mathrm{M}}}\right)$ and 3 additional couplings are introduced: the one between two gluons and the Higgs boson $\left(c_{g}\right)$, the one between 2 gluons and 2 Higgses $\left(c_{2 g}\right)$, and the one between 2 top quarks and 2 Higgses $\left(c_{2}\right)$. A clustering technique [2] has been developed to classify the regions of the EFT 5D parameters space in 12 different shape benchmarks accordingly to their kinematic properties. Several BSM models predict the existence of massive resonances that can decay in pairs of SM Higgs bosons In the following, the presence of a resonance in the di-Higgs invariant mass spectra is studied using the RandallSundrum model described in $[3,4]$ as a benchmark. The analyses presented in these proceedings have been performed at the LHC using the data collected by the CMS experiment with a center of mass energy $\sqrt{s}=13 \mathrm{TeV}$. The data corresponds to a luminosity of 2.3-2.7 $\mathrm{fb}^{-1}$ collected during the 2015 LHC data taking, with the exception of the bb $\tau \tau$ analysis which is performed on $12.9 \mathrm{fb}^{-1}$ data collected in 2016 .

\section{Double Higgs production in the bb $\gamma \gamma$ final state}
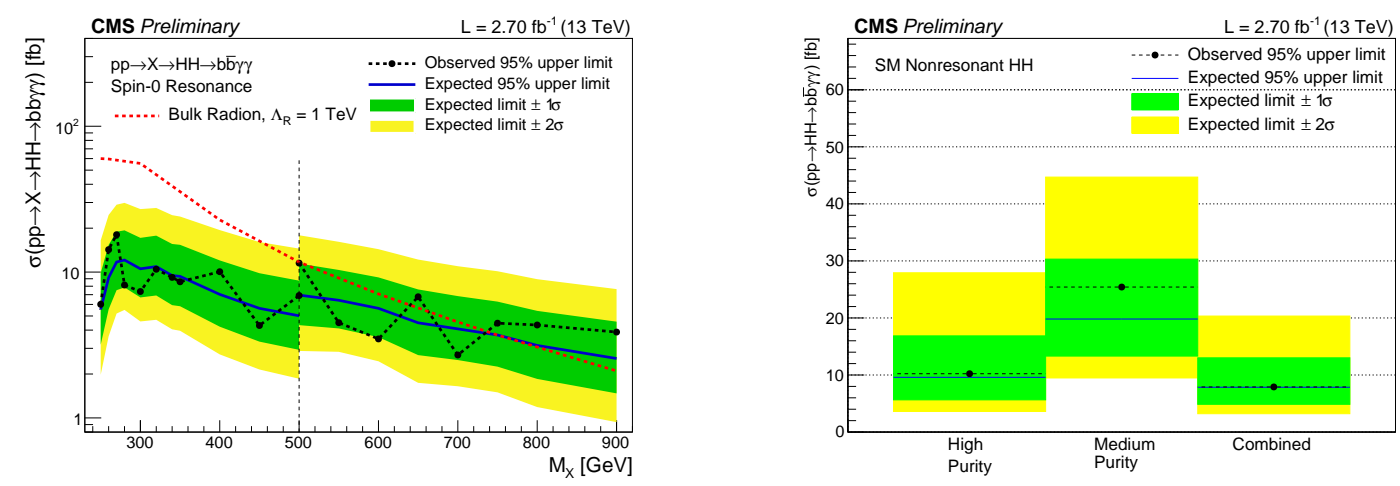

Figure 1: Observed (solid) and expected (dashed) 95\% CL limit on the double Higgs boson resonant (left) and non-resonant (right) production cross-section. The yellow (green) corresponds to the 1 (2) $\sigma$ uncertainty band. The red line shows the expectation for the bulk Radion production for $\Lambda_{\mathrm{R}}=1 \mathrm{TeV}$.

Despite the low branching ratio (BR $h h \rightarrow b b \gamma \gamma=0.26 \%$ ) the bb $\gamma \gamma$ analysis [5] can profit from the excellent CMS resolution on the energy of the photons. Diphoton candidates must have a well identified vertex and the leading (subleading) photon must have $E_{T}>30(20) \mathrm{GeV}, E_{T} / m_{\gamma \gamma}>\frac{1}{3}\left(\frac{1}{4}\right)$ and $100<m_{\gamma \gamma}<180 \mathrm{GeV}$. Dijet candidates are required to have invariant masses of $80<m_{j j}<$ $200 \mathrm{GeV}$. In events with more than two jets, the two most likely to come from $\mathrm{b}$ quark decays accordingly to the Combined Secondary Vertex (CSV) algorithm [6] are selected. The CSV score of the jets is used to divide events in 3 categories, using working points corresponding respectively to $57 \%$ (tight), $78 \%$ (medium) and 95\% (loose) signal efficiency. In the resonant searches, events 
with at least one tight (medium) b-tagged jet are assigned to the High (Medium) Purity category. Loose events are assigned to a control region for the background estimation. In the non-resonant analysis events with both jets passing the medium working point requirement fall in the High Purity category. The variable $\widetilde{M}_{X}=m_{j j \gamma \gamma}-m_{j j}+125 \mathrm{GeV}$ is used in the resonant analysis to define a mass window cut around the resonance mass. In the non-resonant analysis a cut-off of $\widetilde{M}_{X}>350 \mathrm{GeV}$ is applied. The signal extraction is performed fitting the $2 \mathrm{D} m_{j j}, m_{\gamma \gamma}$ distribution of selected events with a Gaussian plus a Crystal-Ball function for the signal and a second order Bernstein function for the background. The limits obtained with these setting are shown in figure 1. Results are dominated by statistical uncertainties, with systematics affecting the results by less than $1 \%$. Non-resonant production is observed to be $<7.85 \mathrm{fb}$, or about 80 times the SM expectation.

\section{Double Higgs production in the bbbb final state}
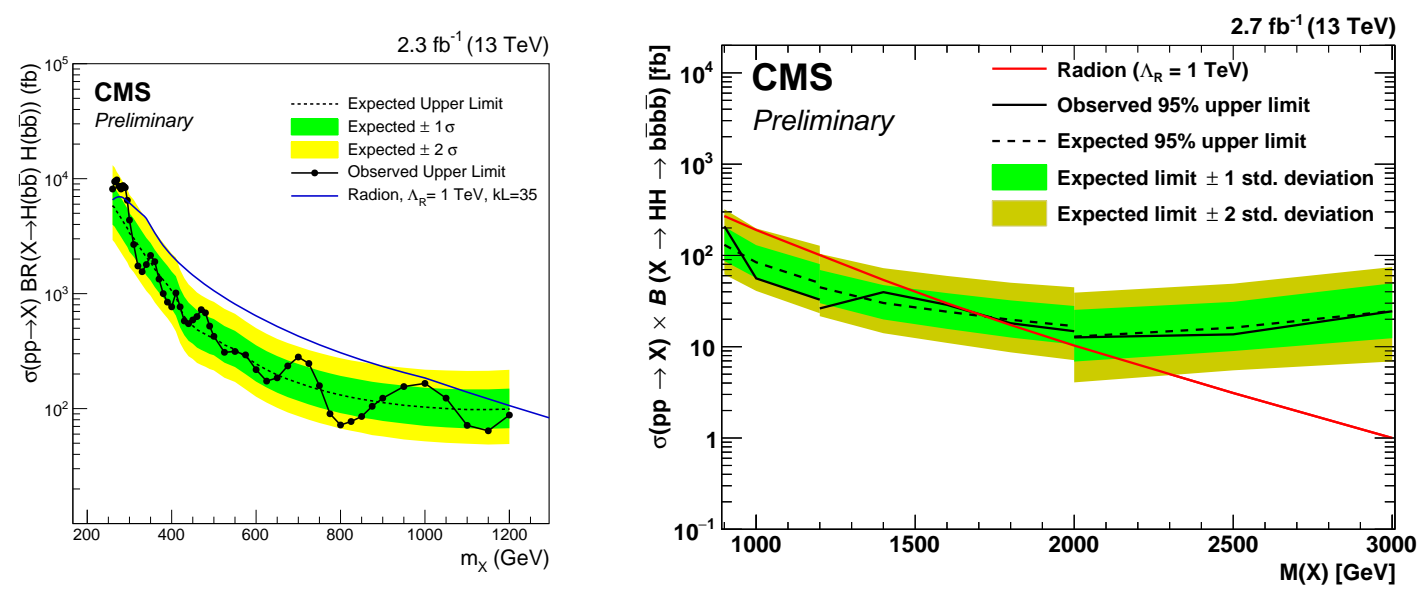

Figure 2: Observed (solid) and expected (dashed) 95\% CL limit on the double Higgs boson resonant production cross-section in the bbbb channel in the LMR and MMR (left) and HMR (right). The blue and red lines show the expectation for the bulk Radion production for $\Lambda_{\mathrm{R}}=1 \mathrm{TeV}$.

The bbbb analysis is divided in 3 mass regions: a Low Mass Ragion (LMR) with $m_{X}<400$ $\mathrm{GeV}$, a Medium Mass Region (MMR, $400<m_{X}<1200 \mathrm{GeV}$ ) and a High Mass Region (HMR) above $1.2 \mathrm{TeV}$, where the jets coming from each Higgs boson are merged due to the high kinematic boost. The non-resonant [7] and resonant analyses in the LMR and MMR [8] rely on events with at least $4 \mathrm{~b}$-tagged jets. The 4 jets with the highest b-tagging score are paired by requesting the two Higgs bosons to have the most similar masses (non-resonant) or minimizing the mass differences with respect to the SM h mass (resonant). Selected events must have dijet invariant masses in the range $50<m_{j j}<300 \mathrm{GeV}$ and applying a Boosted Decision Tree (BDT) to discriminate between signal and background for the non-resonant analysis. In the resonant analysis a circular cut in the $\left(m_{h 1}, m_{h 2}\right)$ distribution is applied and is requested that $\left|m_{j j}-115 \mathrm{GeV}\right|<34 \mathrm{GeV}$ (LMR) or that for each h candidate $\Delta R_{j 1, j 2}<1.5$ (MMR). For the HMR [9] two Higgs tagging algorithms, based on the subjet b-tagging or on an MVA built from the jets kinematics are used to select interesting events. The main background contribution comes from QCD events with multiple jets. It is estimated from data by using the hemisphere mixing technique (non-resonant analysis), from two 
side bands (LMR, MMR), and using the alphabet technique (HMR). The final statistical analysis is performed in the $2 \mathrm{D}\left(m_{h 1}, m_{h 2}\right)$ plane (non-resonant analysis) and on the reconstructed 4-body invariant mass (resonant analysis). The results of the resonant searches are shown in figure 2. The observed (expected) exclusion limit on the non-resonant production cross section corresponds to $3880 \mathrm{fb}$ (3490 fb), or about 300 (280) times the SM production times BR cross-section.

\section{Double Higgs production in the $b b \tau \tau$ final state}

The event selection for the $b b \tau \tau$ analysis $[10,11]$ requires the presence of at least two b-tagged jets and two isolated leptons in the final state. The two jets with the highest CSV score are selected as $\mathrm{b}$ candidates. At least one $\tau$ must have decayed hadronically. Events are categorized according to the $\tau$ decay: $\mu \tau_{h}, e \tau_{h}$, and $\tau_{h} \tau_{h}$ where one $\tau$ decayed into a $\mu$, an $e$, or both decayed in hadrons respectively. An additional boosted category, where the jets originated from the $\tau$ s hadronic decay are merged together is used in the resonant searches to increase the sensitivity at high masses. A veto on the presence of additional isolated leptons is applied to further remove background. The
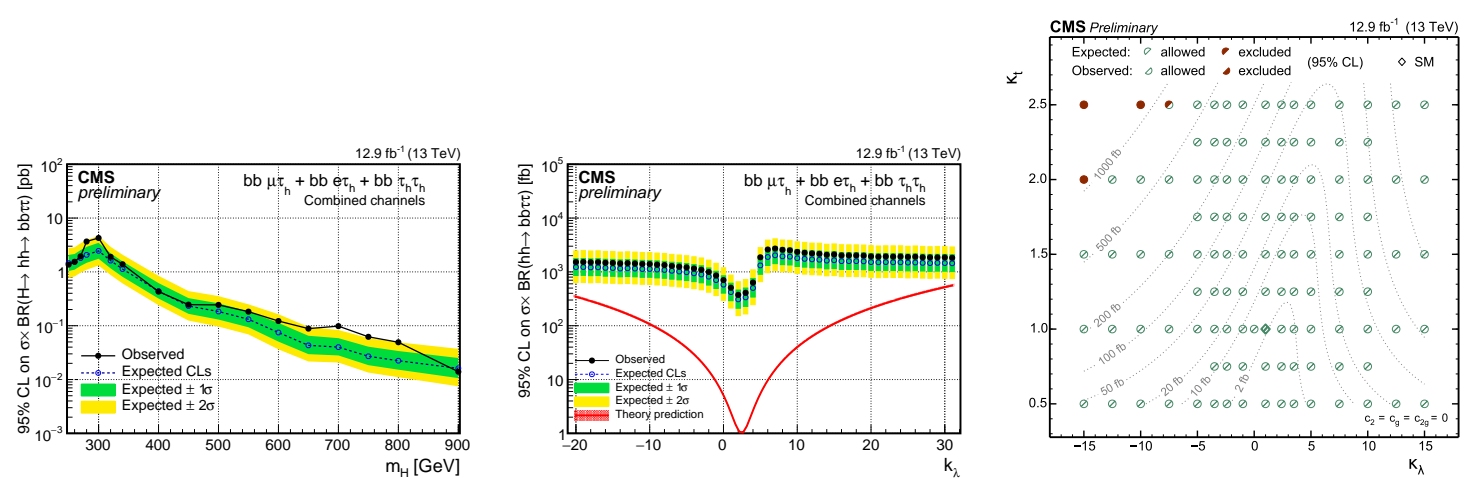

Figure 3: Observed (solid) and Figure 4: Observed (full) and ex-Figure 5: Exclusion of nonexpected (dashed) 95\% CL limit pected (empty) 95\% CL limit on the resonant production as a function on the double Higgs boson reso- double Higgs boson non-resonant of $k_{\lambda}$ and $k_{t}$, fixing the other EFT nant production cross-section in the production cross-section for differ- parameters to 0 . Each full (open) $\mathrm{bb} \tau \tau$ channel. ent values of $k_{\lambda}$. Red line shows the marker denotes a point excluded theory expectations. (compatible) with the current data.

invariant mass of the di-taus system is reconstructed using a likelihood technique [12] and must satisfy $80<m_{\tau \tau}<160 \mathrm{GeV}$. In the resolved categories the di-jet invariant mass is required to be $80<m_{j j}<160 \mathrm{GeV}$, In the boosted category the jet invariant mass has to be in the range $90<$ $m_{j}<160 \mathrm{GeV}$. The main background contributions come from the $t \bar{t}$ and QCD processes. Monte Carlo simulations are used to estimate the shape and expected rates of the $t \bar{t}$. The QCD is evaluated from data using the $\mathrm{ABCD}$ technique from control regions with same charge leptons and relaxed isolation. To reduce the $t \bar{t}$ background contribution in the non-resonant analysis, a BDT built from the angular properties of the final state objects is used to discriminate signal and background events. The visible invariant mass of the $b b \tau \tau$ object is used as discriminating variable in the non-resonant search, while in the resonant searches a kinematic fit [13] of the event is performed. The results are dominated by the statistical uncertainty. Resonant results are shown in figure 3 . The observed exclusion for non-resonant production, shown in figures 4,5 , is $508 \mathrm{fb}\left(200 \times \sigma_{h h \rightarrow b b \tau \tau}^{\mathrm{SM}}\right)$ for $k_{\lambda}=1$. 


\section{Double Higgs production in the bbWW final state}
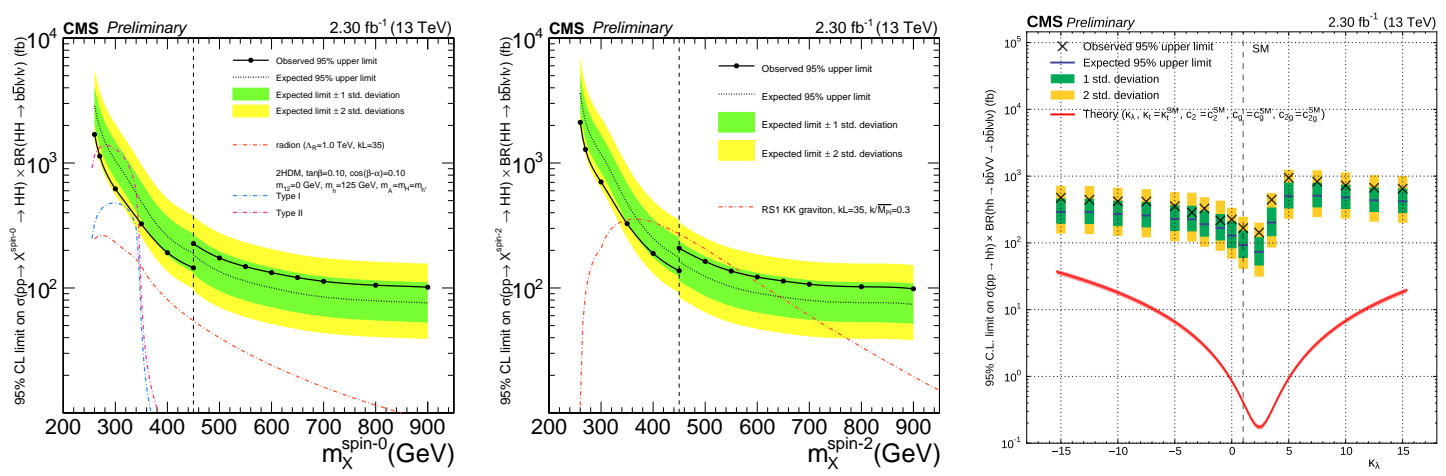

Figure 6: Observed (solid) and expected (dashed) 95\% CL on the double Higgs boson production times BR in the bbWW channel assuming spin-0 (left) or spin-2 (middle) hypotheses compared to theoretical predictions (larger dash). Right: Upper limit on the non-resonant production for different values of $k_{\lambda}$.

This search $[14,15]$ is performed in the decay channel $(X \rightarrow) h h \rightarrow W W \rightarrow l v l v$, with a relatively small (1.23\%) branching fraction. The leading muon (electron) in the event must have $p_{T}>20 \mathrm{GeV}$ and the subleading $p_{T}>10(15) \mathrm{GeV}$, with both having $|\eta|<2.4(2.5)$. Only opposite sign events with dilepton invariant mass $12 \mathrm{GeV}<m_{l l}<m_{Z}-15 \mathrm{GeV}$ are selected. The two jets with the highest CSV score are selected as b candidates, and both of them are required to pass the $70 \%$ efficiency working point. A BDT based on the angles and energies of the decay products and the $E_{T}^{\text {miss }}$ of the event is defined in the non-resonant analysis to separate the signal from the background, with the SM single top, $t \bar{t}$, and Drell-Yan production being the largest sources of background. Two such BDTs are trained for the low $\left(m_{X}<450 \mathrm{GeV}\right)$ and high $\left(m_{X}>450 \mathrm{GeV}\right)$ mass regions in the resonant analysis. To perform the resonant searches the events are divided in 4 categories according to whether the dijet mass is near the Higgs boson mass value $\left(95<m_{j j}<135\right.$ $\mathrm{GeV}$ ) or not and whether the event has a low or high BDT score. In the non-resonant analysis the statistical analysis is performed on the BDT distribution in 3 different regions of $m_{j j}: m_{j j}<75$ $\mathrm{GeV}, 75<m_{j j}<140 \mathrm{GeV}$, and $m_{j j}>140 \mathrm{GeV}$, with the middle one being signal enriched and the other two background dominated. The final results are shown in figure 6. The observed exclusion for the non-resonant production corresponds to $167 \mathrm{fb}$ or about 400 times the SM expectation.

\section{Conclusions}

Results for resonant searches for the presence of spin-0 or spin-2 are summarized in figure 7. Overall, no clear excess is observed in the range $250 \mathrm{GeV}-3 \mathrm{TeV}$. Non resonant searches have been presented, with sensitivities ranging from tens to hundred times the expected SM production.

\section{References}

[1] S. Dawson, A. Ismail, and Ian Low. What's in the loop? The anatomy of double Higgs production. Phys. Rev., D91(11):115008, 2015. 

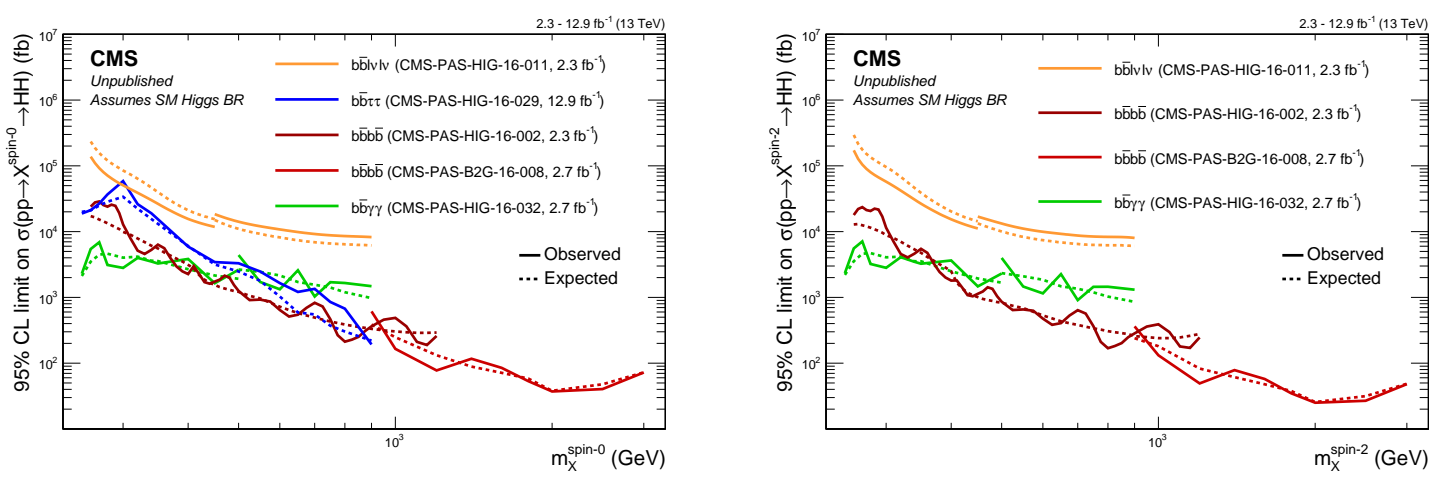

Figure 7: Observed and expected 95\% CL upper limits on the cross section times branching fraction $\sigma(\mathrm{gg} \rightarrow$ $X) \times B R(X \rightarrow H H)$ obtained by the different analyses assuming spin-0 (left) or spin-2 (right) hypotheses.

[2] Alexandra Carvalho, Martino Dall'Osso, Tommaso Dorigo, Florian Goertz, Carlo A. Gottardo, and Mia Tosi. Higgs Pair Production: Choosing Benchmarks With Cluster Analysis. JHEP, 04:126, 2016.

[3] H. Davoudiasl, J. L. Hewett, and T. G. Rizzo. Phenomenology of the Randall-Sundrum Gauge Hierarchy Model. Phys. Rev. Lett., 84:2080, 2000.

[4] Kaustubh Agashe, Hooman Davoudiasl, Gilad Perez, and Amarjit Soni. Warped Gravitons at the LHC and Beyond. Phys. Rev., D76:036006, 2007.

[5] CMS Collaboration. Search for H(bb)H(gammagamma) decays at 13TeV. 2016.

[6] CMS Collaboration. Identification of b quark jets at the CMS Experiment in the LHC Run 2. 2016.

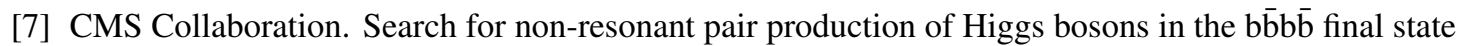
with 13 TeV CMS data. 2016.

[8] CMS Collaboration. Search for resonant pair production of Higgs bosons decaying to two bottom quark-antiquark pairs in proton-proton collisions at $13 \mathrm{TeV} .2016$.

[9] CMS Collaboration. Search for heavy resonances decaying to a pair of Higgs bosons in four b quark final state in proton-proton collisions at $\operatorname{sqrt}(\mathrm{s})=13 \mathrm{TeV} .2016$.

[10] CMS Collaboration. Search for non-resonant Higgs boson pair production in the bbtautau final state using 2016 data. 2016.

[11] CMS Collaboration. Search for resonant Higgs boson pair production in the $b \bar{b} \tau^{+} \tau^{-}$final state using 2016 data. 2016.

[12] Lorenzo Bianchini, John Conway, Evan Klose Friis, and Christian Veelken. Reconstruction of the higgs mass in h to tautau events by dynamical likelihood techniques. Journal of Physics: Conference Series, 513(2):022035, 2014.

[13] Vardan Khachatryan et al. Searches for a heavy scalar boson H decaying to a pair of $125 \mathrm{GeV}$ Higgs bosons hh or for a heavy pseudoscalar boson A decaying to Zh, in the final states with $h \rightarrow \tau \tau$. Phys. Lett., B755:217-244, 2016.

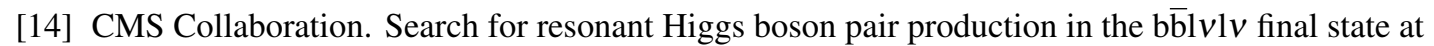
$\sqrt{s}=13 \mathrm{TeV} .2016$.

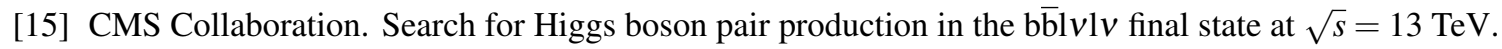
2016. 